\title{
Contaminación mercurial en peces y sedimentos del río Timbiquí, Consejo Comunitario Mayor Renacer Negro, departamento del Cauca
}

\author{
Mercurial contamination in fish and sediments of the Timbiquí River, \\ Major Community Council Renacer Negro, department of Cauca
}

\author{
Fairy María Medina Mosquera'1, Yiskar Damián Murillo Asprilla²
}

\section{Resumen}

Se determinó la contaminación mercurial del río Timbiquí a partir de la medición de mercurio total en muestras de sedimentos y peces. Se encontró que la presencia de este elemento en los sedimentos aun no sobrepasa los límites permisibles para la conservación de la vida acuática establecidos en Canadá $(0,14 \mathrm{mg} / \mathrm{kg})$ y Estados Unidos (0,15 mg/kg); sin embargo, de no tomarse medidas inmediatas estos niveles a mediano y largo plazo podrían aumentar y muy probablemente influir de manera directa en un aumento en el nivel de mercurio en los peces, aunque no todas las muestras registraron niveles por encima de los límites permisibles para consumo humano, sobre todo mujeres embarazadas y niños $(0,2 \mu \mathrm{g} / \mathrm{g})$. Se presentaron niveles importantes que indican que la cuenca está siendo receptora de vertimientos de origen minero con presencia de este metal. Teniendo en cuenta los resultados, se recomienda el diseño e implementación de medidas de manejo ambiental en todas las unidades mineras presentes en el área de influencia del Consejo Comunitario Renacer Negro y el monitoreo periódico de los niveles de mercurio total en las matrices analizadas, tanto en el río Timbiquí como en sus afluentes.

Palabras clave: Mercurio total, Peces, Río Timbiquí, Sedimentos.

\begin{abstract}
The mercurial contamination of the Timbiqui River was determined from the measurement of total mercury in sediments and fish samples. It was found that the presence of this elements in the sediments still does not exceed the permissible limits for the conservation of aquatic life established in Canada (0.14 mg) $\mathrm{kg})$ and the United States $(0.15 \mathrm{mg} / \mathrm{kg})$; however, if actions are not taken immediately, levels could increase in the medium and
\end{abstract}

\author{
Investigadora asociada I, Instituto de \\ Investigaciones Ambientales del Pacífico \\ (IIAAP), Quibdó, Colombia. \\ e-mail: fmedina@iiap.org.co \\ 2 Investigador asociado II, Instituto de \\ Investigaciones Ambientales del Pacífico \\ (IIAAP), Quibdó, Colombia. \\ e-mail: ymurillo@iiap.org.co
}




\section{Bioetnia Volumen 14, 2017}

long term, and very likely influence directlyon the mercury level in the fish, the samples registered levels above the permissible limits for human consumption, especially for pregnant women and children $(0.2 \mu \mathrm{g} / \mathrm{g})$, the study registered important levels that indicate that the basin is receiving mineral discharge with presence of this metal. Based on the results, it is recommended a design and implementation of an environmental management strategy in all the mining units present in the area of influence of the Major Community Council Renacer Negro, and the periodic monitoring of the total mercury levels in the matrices analyzed, both in the river Timbiqui as in its tributaries.

Keywords: Fish, Sediments, Timbiquí River, Total mercury.

\section{Introducción}

La actividad minera de oro y platino en la cuenca del río Timbiquí, Colombia, está ocasionando un deterioro en la calidad del agua, sobre todo por su uso como receptor de vertimientos mineros, que por lo general llegan a las fuentes hídricas sin la aplicación de medidas que reduzcan las cargas contaminantes procedentes del lavado del material aluvial en el proceso de extracción del metal. De igual forma, ocasiona la sedimentación de los cuerpos de agua, alteración de los cauces, erosión de las orillas y en algunos casos disminución del recurso ictiológico, convirtiendo a esta actividad en una práctica insostenible que afecta negativamente los componentes del ecosistema a nivel local y regional.

El río Timbiquí, principal fuente de consumo, transporte y comunicación de las comunidades del municipio de Santa Bárbara de Timbiquí, y de los centros poblados presentes en sus riberas, en la actualidad se encuentra fuertemente intervenido por el desarrollo de procesos de extracción minera, los cuales están distribuidos no solo sobre su cauce principal, sino también sobre sus afluentes, lo que puede ocasionar la alteración de las características fisicoquímicas y microbiológicas del agua y limitación de su uso en otras actividades asociadas con el recurso como el consumo humano y uso doméstico, la recreación, la conservación de especies acuáticas y en especial el uso del recurso para la pesca. De igual manera se están generando riesgos de salubridad pública relacionados (enfermedades de tipo gastrointestinal y de tipo toxicológico), con la presencia de organismos patógenos y sustancias tóxicas proveniente de los efluentes mineros y de las actividades domésticas desarrolladas en los campamentos como el mercurio.

La actividad minera está contribuyendo con el aporte de mercurio en el medio ambiente y por consiguiente con su incorporación en la cadena alimenticia a través de procesos biológicos que llegan hasta el hombre, como consumidor final. El mercurio se acumula en sedimentos en la base de los cuerpos de agua, donde los microorganismos como las bacterias que viven allí pueden convertirlo a la forma orgánica del metilmercurio, que es sacado por los gusanos y otros animales pequeños que viven en los sedimentos; este compuesto se acumula en los peces que comen estos animales y en los peces más grandes que comen a los más pequeños (Mancera-Rodríguez y Álvarez-León 2006). Según Ramos et al. (2000) 40\% del mercurio contenido en peces, se bioacumula en forma de metilmercurio quedando disponible hasta llegar al hombre por medio de la cadena trófica.

El mercurio es un metal considerado altamente tóxico para la salud humana, pudiendo desarrollar alteraciones en las funciones del sistema nervioso, el cual es esencialmente sensible al metilmercurio con consecuencias neurofisiológicas, sobre todo en el desarrollo de los fetos y en los niños (Lebel et al. 1997).

Según Mancera-Rodríguez y Álvarez-León (2006), la contaminación por mercurio y otros metales pesados es muy difícil de detectar a través del monitoreo medioambiental, debido 
a que las técnicas de análisis son específicas y costosas, además las concentraciones en el medio suelen ser más bajas que las encontradas en los sedimentos, o en las especies de fauna y flora presentes en los cuerpos de agua; por esto, en ocasiones, una baja concentración en la columna de agua no necesariamente indica baja contaminación o poca utilización del elemento. Teniendo en cuenta lo anterior y la falta de información suficiente que muestre los niveles de afectación en las características físicas, químicas y biológicas de las fuentes hídricas receptoras de vertimientos mineros, resulta interesante evaluary analizar los niveles de afectación y el comportamiento del mercurio sobre el recurso íctico, y la salud humana, de manera que se pueda hacer una identificación de las principales afectaciones ambientales que se están presentando en la zona por este tipo contaminación. Por lo tanto, se plantea la siguiente pregunta de investigación: ¿Cuál es el nivel de mercurio que presentan los peces y los sedimentos del río Timbiquí, municipio de Santa Bárbara de Timbiquí, departamento del Cauca?

\section{Metología}

Área de estudio. El municipio de Timbiquí, está situado en el suroeste de Colombia, entre las regiones andina y pacífica y hace parte del denominado Chocó Biogeográfico, caracterizado por ser una de las regiones más húmedas y con gran biodiversidad; está lo- calizado entre los $00^{\circ} 58^{\prime} 54^{\prime \prime}$ y $03^{\circ} 19^{\prime} 04^{\prime \prime} \mathrm{N}$ y los $75^{\circ} 47^{\prime} 36^{\prime \prime}$ y $77^{\circ} 57^{\prime} 05^{\prime \prime}$ W. Cuenta con una extensión de $1.813 \mathrm{~km}^{2}$ y una altura de $5 \mathrm{msnm}$, una temperatura de $28^{\circ} \mathrm{C}$, una pluviosidad de $6.000 \mathrm{~mm}$ anuales y la humedad relativa oscila alrededor de $93 \%$ originada por las altas corrientes marinas. Se localiza al occidente del departamento del Cauca en la costa pacífica, a una distancia de $580 \mathrm{~km}^{2}$ de Popayán, la capital. La topografía del municipio es quebrada en un $70 \%$, con presencia de algunas ramificaciones montañosas y planicie; cuenta con 26 corregimientos y de acuerdo a su hidrología, tiene 3 fuentes hídricas principales, el río Timbiquí, el río Saija y el río Bubuey (municipio de Timbiquí 2012-2015 PBOT) (Figura 1)

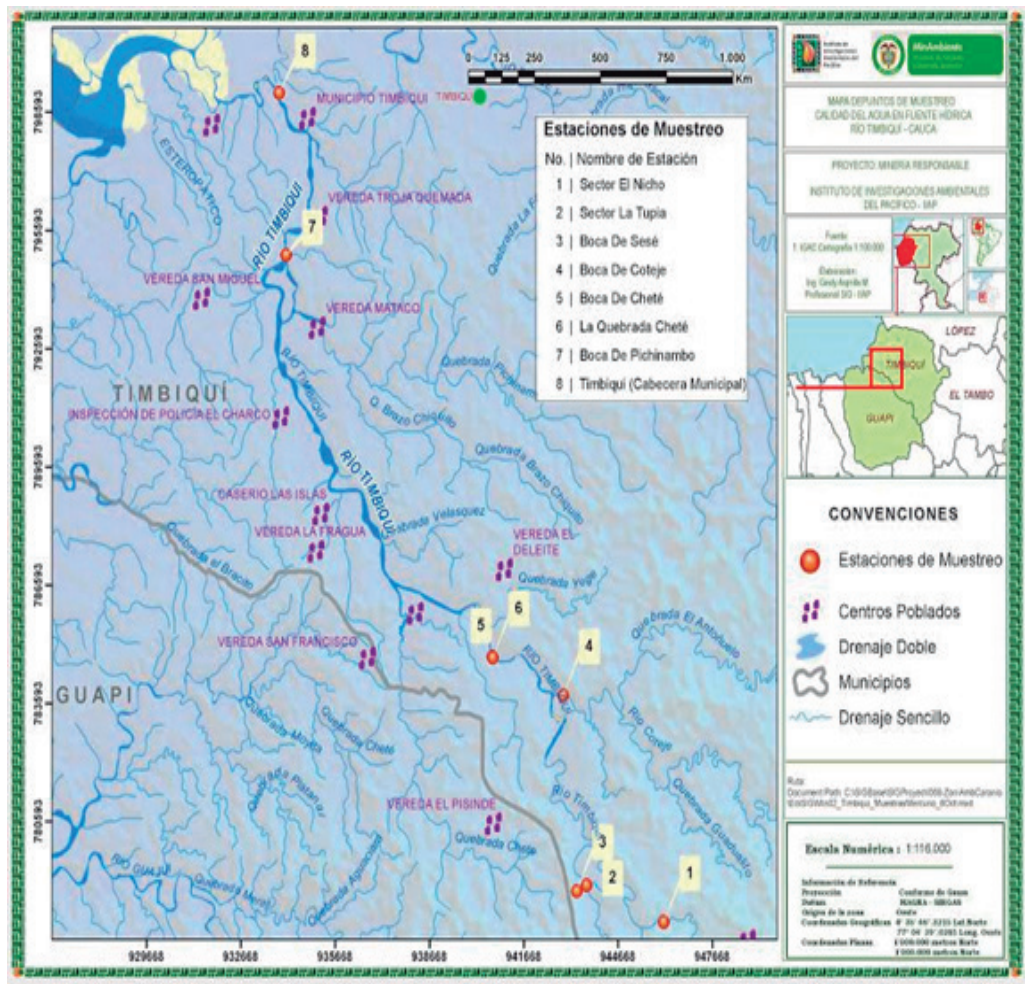

Figura 1. Localización del área de estudio y puntos de muestreo.

Los principales afluentes del río Timbiquí son: los ríos Chete, Coteje y Sesé, y la quebrada Pichinambó. El río Timbiquí, junto con el río Saija, son los de mayor longitud en el municipio y es navegable todo el tiempo hasta Santa Bárbara de Timbiquí (cabecera municipal). La minería es una de las fuentes de ingreso más importante para las comunidades aledañas a las fuentes hídricas del municipio ((municipio de Timbiquí 20122015 PBOT).

La cuenca del río Timbiquí, en el momento del muestreo, 


\section{Bioetnia Volumen 14, 2017}

presentó agua claras en la parte alta y en algunos sectores comprendidos por las desembocaduras de los efluentes estudiados, estaban turbias, producto posible de la presencia de vertimientos mineros. Esta cuenca presenta sustrato compuesto por arena y piedras de regular tamaño. Se registraron altas concentraciones de oxígeno disuelto, buenas condiciones de temperatura y en condiciones generales, bajas concentraciones de sólidos suspendidos y turbiedad (Figura 2, Tabla 1).

\section{Método}

Análisis de mercurio en muestras de sedimentos. Para analizar los niveles de mercurio en muestras de sedimentos del río Timbiquí, dadas las condiciones hidráulicas del cauce principal, de manera manual se tomaron aproximadamente un $\mathrm{kg}$ de sedimentos por punto de muestreo. Estas muestras fueron almacenadas y refrigeradas en bolsas herméticas, hasta su llegada al laboratorio del Grupo GAIA de la Universidad de Antioquia, Medellín, Colombia, donde se realizó la detención de mercurio total (HgT).

Análisis de mercurio en muestras de peces. Los peces se capturaron directamente por pescadores locales. Para la determinación taxonómica de los ejemplares, se utilizaron claves, listados, descrip- ciones y figuras, descritas en: Román-Valencia (1988, 1990), Cala (1990), Rivas-Lara (1993), Lasso et al. (2011), Maldonado-Ocampo et al. (2005, 2012), Jiménez-Segura et al. (2014).Acada individuo se le tomaron las medidas biométricas, longitud total (LT) y peso total (W). Luego se depositaron en bolsas plásticas con sus respectivos datos de campo (fecha y lugar de captura, nombre científico y nombre común,) y se almacenaron en una nevera con abundante hielo. Con ayuda de un elemento corto pulsantes esterilizado, a cada espécimen se le extrajo una muestra del músculo de la parte posterior de la aleta dorsal. Estas submuestras se depositaron individualmente en frascos plásticos esterilizados y rotulados. Por último, se enviaron totalmente congeladas al Laboratorio de Análisis GAIA, donde se realizó la detención de mercurio total (HgT) (Figura 3).

El resultado del análisis para cada muestra se presenta como el promedio $( \pm)$ y la desviación estándar (DE) de las muestras que superaron los dos individuos analizados. Para determinar diferencias significativas, se utilizó una prueba no paramétrica de Kruskall Wallis. Todo lo anterior se hizo con ayuda del software estadístico Infostat versión 2016.

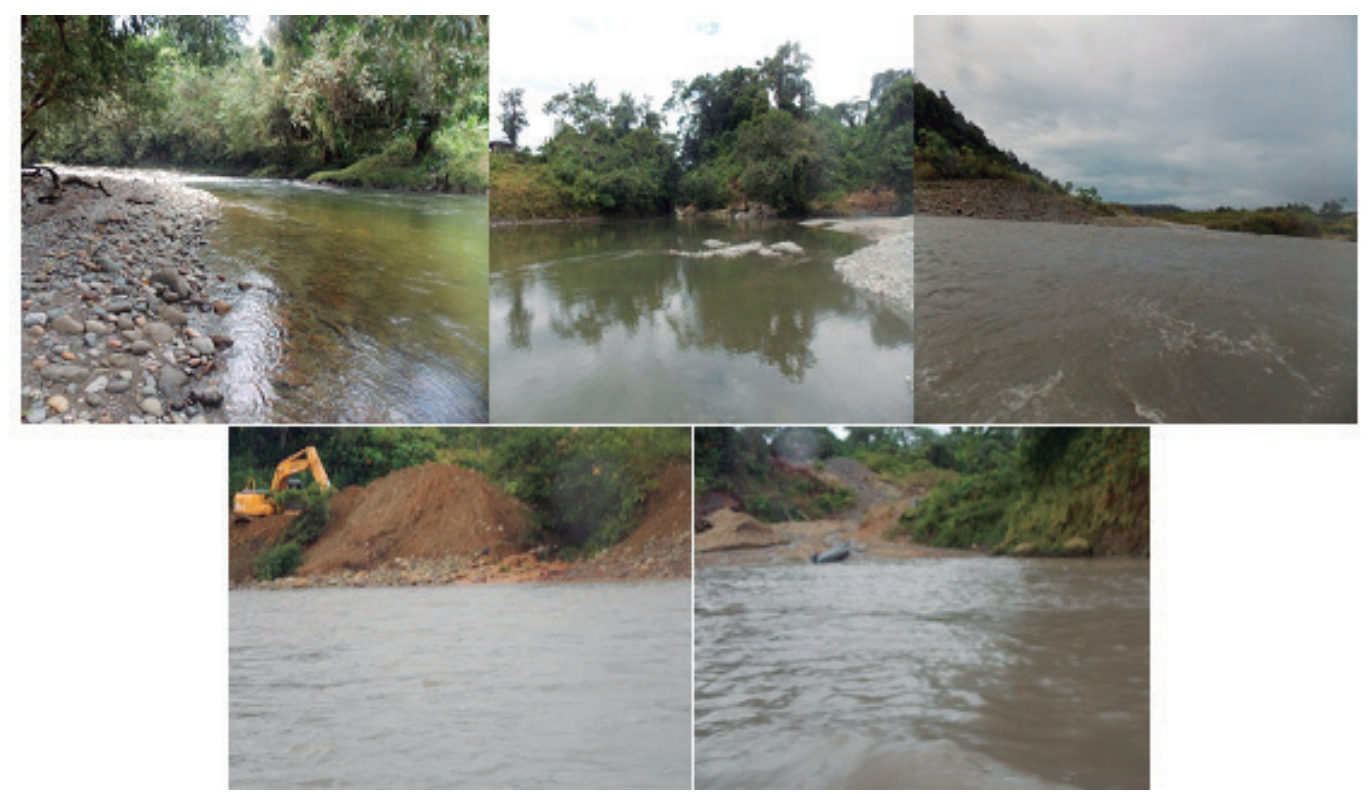

Figura 2. Puntos de muestreo en la cuenca del río Timbiquí. 
Mercurio en río Timbiquí. FM Medina Mosquera, YD Murillo

Tabla 1. Puntos de muestreo cuenca del río Timbiquí

\begin{tabular}{|c|c|c|}
\hline Puntos & Descripción & Coordenadas \\
\hline 1 & Río Timbiquí sector El Nicho & $\mathrm{N}=02^{\circ} 35^{\prime} 19,2^{\prime \prime} \mathrm{W}=077^{\circ} 33^{\prime} 43.2^{\prime \prime}$ \\
\hline 2 & Río Timbiquí sector La Tupia & $\mathrm{N}=02^{\circ} 35^{\prime} 49,9^{\prime \prime} \mathrm{W}=077^{\circ} 35^{\prime} 02.7^{\prime \prime}$ \\
\hline 3 & Boca de Sesé & $\mathrm{N}=02^{\circ} 35^{\prime} 44,9^{\prime \prime} \mathrm{W}=077^{\circ} 35^{\prime} 12.7^{\prime \prime}$ \\
\hline 4 & Boca de Coteje & $\mathrm{N}=02^{\circ} 38^{\prime} 26,8^{\prime \prime} \mathrm{W}=077^{\circ} 35^{\prime} 26.7^{\prime \prime}$ \\
\hline 5 & Río Timbiquí aguas arriba boca de Cheté & $\mathrm{N}=02^{\circ} 38^{\prime} 59,0^{\prime \prime} \mathrm{W}=077^{\circ} 36^{\prime} 40.0^{\prime \prime}$ \\
\hline 6 & Boca de la quebrada Cheté & $\mathrm{N}=02^{\circ} 38^{\prime} 58.1^{\prime \prime} \mathrm{W}=077^{\circ} 36^{\prime} 39.4^{\prime \prime}$ \\
\hline 7 & Río Timbiquí aguas arriba boca de Pichinambo & $\mathrm{N}=02^{\circ} 44^{\prime} 30,7^{\prime \prime} \mathrm{W}=077^{\circ} 40^{\prime} 12^{\prime \prime}$ \\
\hline 8 & $\begin{array}{l}\text { Río Timbiquí aguas abajo de Santa Rosa de Timbiquí } \\
\text { (cabera municipal) }\end{array}$ & $\mathrm{N}=02^{\circ} 46^{\prime} 44.6^{\prime \prime} \mathrm{W}=077^{\circ} 40^{\prime} 19.3^{\prime \prime}$ \\
\hline
\end{tabular}

\section{Resultados y discusión}

Composición íctica. Se capturó en total 85 individuos de las cuales se tomaron 35 agrupados en 8 familia y 11 especies ícticas para los correspondientes análisis de mercurio. La especie más abundante fue: Chaetestona $s p$. con 12 organismos; los otros 23 se repartieron entre las especies restantes con valores que no superan los cuatros individuos. En la Tabla 2 se resumen los resultados obtenidos del número de individuos por especie.

Análisis del contenido de mercurio total (HgT) en tejido

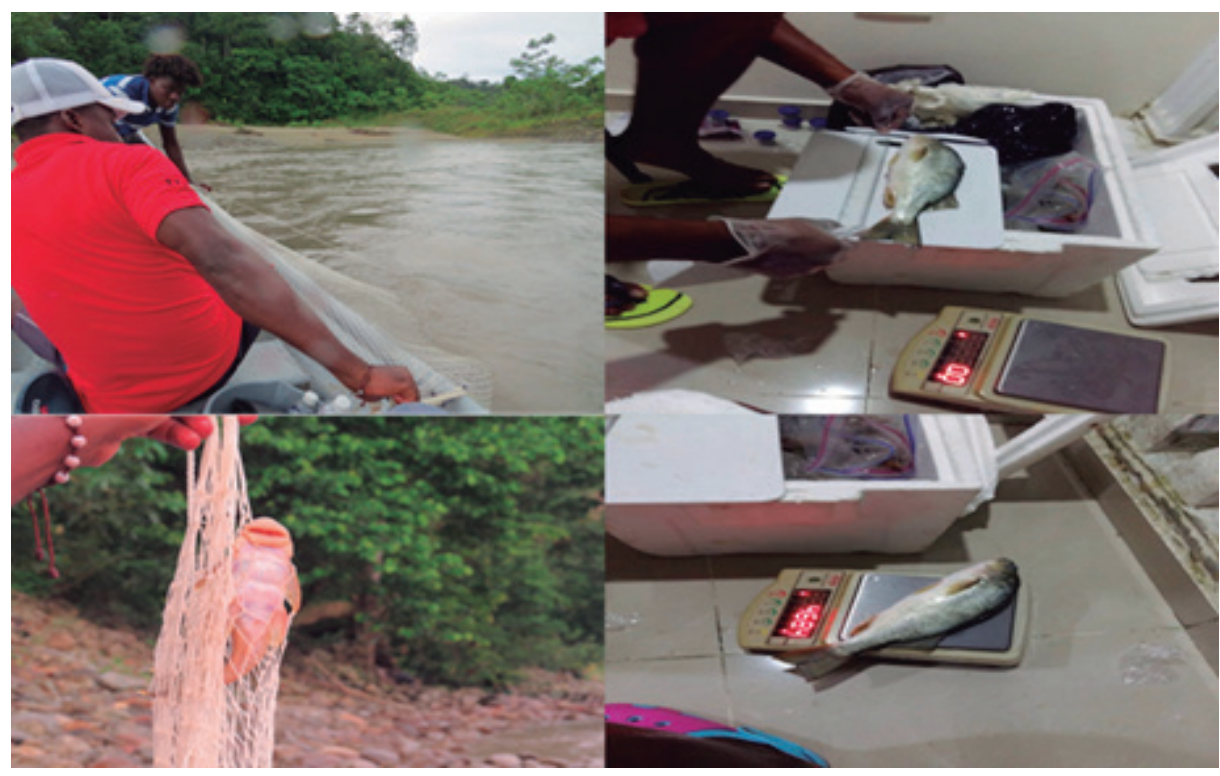

Figura 3. Método para el levantamiento y proceso de los datos tomados en campo. Captura de peces con ayuda de pescadores locales y toma de medidas biométricas.

muscular depeces. El análisis de la concentración de mercurio se realizó en 32 peces, debido a que tres ( 2 guacucos y un sábalo) no presentaron las características biométricas (longitud total y peso) necesarios para la terminación de $\mathrm{HgT}$, según el laboratorio encargado de los análisis. En la Tabla 3 se muestran los resultados obtenidos en el análisis de mercurio, el promedio (Prom), desviación estándar(DE), valores máximos (Máx)y mínimos (Mín) de mercurio encontrado en músculos de las diferentes especies capturadas.

Las concentraciones de mercurio para el total de valor promedio de $1.610 \pm 0,83 \mathrm{mg} / \mathrm{kg}$ con un valor mínimo de 0.820 y máximo de $2.500 \mathrm{mg} / \mathrm{kg}$ (Tabla 3). Las concentraciones de Hg en Cathorops $s p$. fueron altas $(0,700 \mathrm{mg} / \mathrm{kg})$ en comparación con las concentraciones promedio de las otras especies, las cuales exhibieron promedios inferiores de $0,330 \mathrm{mg} / \mathrm{kg}$ de $\mathrm{Hg}$. Este resultado evidencia la existencia de mercurio en la biota estudiada. Además, muestra casi una tendencia del patrón de bioacumulación del metal en la fauna íctica a medida que se asciende en la cadena trófica de este las muestras de tejido muscular analizadas, tuvo un 


\section{Bioetnia Volumen 14, 2017}

Tabla 2. Especies capturadas y número de individuos registrado en el río Timbiquí, departamento del Cauca, Colombia

\begin{tabular}{|c|c|c|c|}
\hline \multirow[t]{2}{*}{ Familia } & \multicolumn{2}{|c|}{ Nombre } & \multirow[t]{2}{*}{$\mathbf{N}^{\circ}$ individuos } \\
\hline & regional & científico & \\
\hline Heptapteridae & Barbudo & Rhamdia quelen & 4 \\
\hline Gobiidae & Bocon & Awaous banana & 1 \\
\hline Ariidae & Chivo & Cathorops sp. & 4 \\
\hline Cetopsidae & Doncella & Trichomycterus sp. & 2 \\
\hline \multirow{2}{*}{ Loricariidae } & Guacuco & Chaetostoma sp. & 12 \\
\hline & Chuchulapa & Rineloricaria sp. & 1 \\
\hline Erytrinidae & Quicharo & Hoplias sp. & 1 \\
\hline \multirow{3}{*}{ Characidae } & Sabaleta & Brycon sp. & 4 \\
\hline & Sabalo & Brycon sp1. & 1 \\
\hline & Sardina & Astyanax sp. & 4 \\
\hline Haemulidae & Cubo & Pomadasys bayanus & 1 \\
\hline
\end{tabular}

Tabla 3. Medidas resumen de las concentraciones de $\mathrm{Hg}$ de las especies analizadas

\begin{tabular}{lrcccc}
\hline Nombre científico & $\mathbf{n}$ & Prom & DE & Mín & Máx \\
\hline Astyanax $s p$ & 4 & 0,330 & 0,120 & 0,200 & 0,490 \\
Brycon $s p$ & 4 & 0,140 & 0,080 & 0,070 & 0,250 \\
Cathorops $s p$ & 4 & 0,700 & 0,260 & 0,370 & 0,920 \\
Chaetostoma $s p$ & 10 & 0,100 & 0,050 & 0,060 & 0,190 \\
Rhamdia quelen & 4 & 0,120 & 0,110 & 0,050 & 0,280 \\
Trichomycterus sp & 2 & 0,220 & 0,210 & 0,070 & 0,370 \\
$\Sigma$ & 28 & 1,610 & 0,830 & 0,820 & 2,500 \\
\hline
\end{tabular}

Variable=Concentración $(\mathrm{mg} / \mathrm{kg})$

grupo, donde a pesar de no encontrarse diferencia estadística significativa, las especies herbívoras presentaron las concentraciones más bajas y las omnívoras junto con las carnívoras mostraron una mayor acumulación. En sintonía con este reporte, algunos investigadores como Ruiz et al. (1996), Ramos et al. (2000), Cala (2001), Olivero y Johnson (2002), Olivero et al. (2015), Cobaleda (2005), Marrugo et al. (2007, 2008), Álvarez (2012), Lancheros-Ascencio (2013), IIAP(2014), encontraron las concentraciones de mercurio más altas en especies como Hoplias malabaricus, Ageneiosus caucanusy Pseudopimelodus schulzi, que se encuentran en la parte alta de la cadena alimenticia, como también sucede con las especies de mayor concentración de este estudio.

Comparación espacial entre los valores de HgT por especie. De acuerdo con el análisis de varianza de modelo mixto, se observó que las medias de los puntos 1 y 2 no son significativamente diferentes (Tabla 4). Sin embargo, es importante resaltar, que la presencia de HgT en los organismos del punto 1 (sitio sin minería referente) obedece quizás al comportamiento de migración local de las especies registradas en esta área, porque les permite desplazarse constantemente a lo largo y ancho de todo el sistema hídrico, en especial en el proceso de receso de la actividad minera (punto 
Tabla 4. Medias de HgT entre los puntos estudiados

\begin{tabular}{cccc}
\hline Sitios & Medias & DE & \\
\hline Punto 1 & 0,18 & 0,29 & A \\
Punto 2 & 0,18 & 0,16 & A \\
\hline
\end{tabular}

2 , en condiciones fisicoquímicas mejoradas por la no actividad), la cual les facilita la actividad de forrajeo, donde posiblemente estén involucrando elementos alimenticios contaminados con $\mathrm{HgT}$ (insectos acuático, larvas, peces de menor tallas, crustáceos, algas, etc.); una vez terminada su actividad, es probable que estos organismos retomen su hábitat (punto 1). De otro lado, se aclara que los valore de $\mathrm{HgT}$ encontrado en las especies del punto 1 , no superan $0,5 \mathrm{mg} / \mathrm{kg}$ permitidos para el consumo de peces con mercurio, mientras tanto algunas están por encima de los $0,2 \mathrm{ug} / \mathrm{g}$ los límites aceptados nacionales (Tabla 5).

Por otro lado, es importante resaltar que $87,5 \%$ de las muestras analizadas presentaron una concentración de $\mathrm{HgT}$ inferior a $0,5 \mathrm{mg} / \mathrm{kg}$, y solo $12,5 \%$, mostró una concentración superior al límite permisible para el consumo humano de la población en general (Resolución $\mathrm{N}^{\circ} 000122$ de 2012 del Ministerio de Salud y Protección Social de Colombia). Sin embargo, al comparar los resultados de HgT encontrados con la norma WHO (2000), se observa que $34,4 \%$ está por encima de $0,2 \mu \mathrm{g} / \mathrm{g}$ permisible para el consumo de pescado con mercurio para las poblaciones vulnerable (menores, mujeres embarazadas y lactantes).

De esto queda por decir, que aunque no se haya encontrado un alto porcentaje de individuos por encima de lo estipulado en las normas nacionales e internacionales, estos resultados no dejan de ser preocupantes, debido a la importancia de consumo que tiene la fauna íctica para las comunidades ribereñas del río Timbiquí y sus afluentes, de donde obtienen gran proteína, ácidos grasos y vitamina D. Como es importante tener claro que el consumo de las especies ícticas con alto contenido de $\mathrm{Hg}$ aumentan las posibilidades de contaminación, direccionando un efecto en la salud de las personas que consumen con mayor frecuencia especies de peces de hábitos carnívoros u omnívoros con tendencia carnívoras como el cubo (Pomadasys bayanus) el chivo (Cathorops sp.), que fueron las especies con mayor concentración de mercurio en esta investigación.

Análisis de mercurio en sedimentos. Los resultados de las concentraciones de mercurio en las muestras de sedimentos superficiales tomadas sobre el cauce principal del río Timbiquí aparecen en la Tabla 6. Las concentraciones más bajas $(0,8$ $\mathrm{mgHg} / \mathrm{kg}$ ) se registraron en las muestras de sedimentos tomadas en los puntos 1 y 3 , los cuales corresponden al sitio de referencia o sin presencia de actividad minera y a la boca de la quebrada Sesé. La concentración más alta $(0,13 \mathrm{mgHg} / \mathrm{kg})$ se registró en la muestra de sedimentos tomada en el punto 5, ubicado sobre el cauce principal del río Timbiquí aguas arriba de la boca de la quebrada Cheté, lugar donde además se registraron las concentraciones más altas de sólidos suspendidos totales y de turbiedad, producto posiblemente por la presencia de vertimientos directos de mineros y sin la aplicación de tratamientos que reduzcan la carga contaminante proveniente del lavado del material aluvial.

Ninguna de las muestras analizadas sobrepasaron los límites permisibles para la conservación de la vida acuática establecidas por Canadá $(0,14$ $\mathrm{mg} / \mathrm{kg}$ ) y Estados Unidos $(0,15 \mathrm{mg} / \mathrm{kg}$ ) (Gaudet et al. 2005); sin embargo, es importante mencionar que de no tomarse medidas de manera inmediata, probablemente a largo plazo se podrían generar problemas ambientales, con repercusión en la fauna acuática principalmente en lospeces, matriz analizada con algunos registros de mercurio por encima de los límites permisibles para consumo humano $(0,2 \mathrm{mg} / \mathrm{kg})$ en población vulnerable (niños y mujeres en embarazo) e inclusive para la población en general $(0,5 \mathrm{mg} / \mathrm{kg})$. Lo anterior genera un riesgo potencial de contaminación 
Bioetnia Volumen 14, 2017

Tabla 5. Niveles de mercurio encontrado en las 32 muestras de músculo de peces analizadas. Las especies de color rojo superan la normatividad nacional

\begin{tabular}{|c|c|c|c|c|}
\hline \multirow[t]{2}{*}{ Sitios } & \multicolumn{2}{|c|}{ Nombre } & \multirow[t]{2}{*}{ Gremios } & \multirow[t]{2}{*}{$\mathrm{HgT}(\mathrm{mg} / \mathrm{kg})^{\mathrm{a}, \mathrm{b}}$} \\
\hline & regional & científico & & \\
\hline \multirow{10}{*}{ Punto 1} & Sabaleta & Brycon sp. & Omnívora & 0,252 \\
\hline & Sabaleta & Brycon sp. & Omnívora & 0,112 \\
\hline & Sabaleta & Brycon sp. & Omnívora & 0,142 \\
\hline & Sabaleta & Brycon sp. & Omnívora & 0,071 \\
\hline & Sardina & Astyanax sp. & Omnívora & 0,360 \\
\hline & Sardina & Astyanax sp. & Omnívora & 0,281 \\
\hline & Sardina & Astyanax sp. & Omnívora & 0,200 \\
\hline & Sardina & Astyanax sp. & Omnívora & 0,491 \\
\hline & Guacuco & Chaetostoma sp. & Omnívora & 0,151 \\
\hline & Guacuco & Chaetostoma sp. & Herbívora & 0,071 \\
\hline \multirow{22}{*}{ Punto 2} & Barbudo & Rhamdia quelen & Omnívora & 0,096 \\
\hline & Barbudo & Rhamdia quelen & Omnívora & 0,282 \\
\hline & Barbudo & Rhamdia quelen & Omnívora & 0,052 \\
\hline & Barbudo & Rhamdia quelen & Omnívora & 0,069 \\
\hline & Doncella & Trichomycterus sp. & Insectívora & 0,369 \\
\hline & Doncella & Trichomycterus sp. & Insectívora & 0,071 \\
\hline & Bocon & Awaous banana & Omnívora & 0,075 \\
\hline & Guacuco & Chaetostoma sp. & Herbívora & 0,091 \\
\hline & Guacuco & Chaetostoma sp. & Herbívora & 0,073 \\
\hline & Guacuco & Chaetostoma sp. & Herbívora & 0,142 \\
\hline & Chivo & Cathorops sp. & Omnívora & 0,365 \\
\hline & Chivo & Cathorops sp. & Omnívora & 0,922 \\
\hline & Chivo & Cathorops sp. & Omnivora & 0,898 \\
\hline & Chivo & Cathorops sp. & Omnívora & 0,611 \\
\hline & Quicharo & Hoplias sp. & Carnívora & 0,100 \\
\hline & Guacuco & Chaetostoma sp. & Herbívora & 0,055 \\
\hline & Guacuco & Chaetostoma sp. & Herbívora & 0,076 \\
\hline & Guacuco & Chaetostoma sp. & Herbívora & 0,191 \\
\hline & Guacuco & Chaetostoma sp. & Herbívora & 0,069 \\
\hline & Guacuco & Chaetostoma sp. & Herbívora & 0,059 \\
\hline & Chuchulapa & Rineloricaria sp. & Herbívora & 0,070 \\
\hline & Cubo & Pomadasys bayanus & Carnívora & 0,611 \\
\hline
\end{tabular}

${ }^{a}$ Normatividad nacional $=0,5 \mathrm{mg} / \mathrm{kg} \quad{ }^{\mathrm{b}} \mathrm{WHO} 2000=0,2 \mu \mathrm{g} / \mathrm{g}$

mercurial por consumo de pescado principalmente Consideraciones finales

a mediano y largo plazo por parte de las comu-

nidades aledañas al río Timbiquí, lugar donde se

registraron los niveles más altos de mercurio en las muestras de sedimentos.

En el Consejo Comunitario de Renacer Negros, los impactos ambientales generados sobre las fuentes hídricas superficiales por la actividad 
Tabla 6. Resultados de los análisis de mercurio en muestras de sedimentos provenientes del río Timbiquí

\section{Punto de muestreo Concentración $(\mathrm{mgHg} / \mathrm{kg})$}

\begin{tabular}{ll}
\hline P1 & 0,08 \\
P2 & 0,10 \\
P3 & 0,08 \\
P4 & 0,10 \\
P5 & 0,13 \\
P6 & 0,09 \\
P7 & 0,12 \\
\hline
\end{tabular}

minera a cielo abierto, son el resultado de la inadecuada utilización de sustancias tóxicas, la falta de implementación de tecnologías limpias durante el proceso, conciencia ambiental de los mineros, control ambiental por parte del estado (instituciones ambientales y minera) e implementación de medidas de manejo ambiental para la utilización del recurso agua. La manera como es extraído el metal en suelos aluviales, hace que se presente una alta demanda del recurso agua, razón por la cual se generan grandes volúmenes de efluentes mineros los cuales son dispuestos inadecuadamente en los cuerpos de aguas superficiales, ocasionando la alteración de las condiciones fisicoquímicas naturales de las fuentes hídricas receptoras, lo que reduce su uso en diferentes actividades sin que ello represente un riesgo para la salud humana (sobre todo por la contaminación mercurial) y para el desarrollo de los procesos hidrobiológicos. Por otro lado, el aporte de altos contenidos de sedimentos y otros contaminantes, ocasionan entre otros aspectos, la sedimentación de los cuerpos de aguas y con ello la reducción del recurso ictiológico y la vida acuática en general, aumenta las probabilidades de ocurrencia de inundaciones y afectan la navegabilidad, principal medio de transporte y de comunicación de la zona.

Lo anterior, hace necesaria y urgente la implementación de medidas de manejo durante el desarrollo de los procesos de extracción minera, que contribuyan a la reducción de las cargas contaminantes generadas en el lavado de la grava e impedir su llegada de manera directa a los cuerpos de aguas, sobre todo si contienen mercurio. También se deben implementar monitoreos periódicos de las descargas antes de su vertimiento, garantizando así que el agua cumpla con los criterios de calidad para vertimientos establecidos por el Decreto 1594/94.

\section{Literatura citada}

Álvarez S. 2013. Acumulación de mercurio (Hg) en tejido muscular y hepático en especies ícticas en diferentes ciénagas del Magdalena medio. Medellín: Universidad de Antioquia, Corporación Académica Ambiental Maestría en Ciencias Ambientales. pp. 20-4.

Cala P. 1990. Diversidad, adaptación ecología y distribución geográfica de las familias de peces de aguadulce de Colombia. Rev Acad Colomb Cienc Ex Fis Nat. 17 (67): 720-40.

Cala P. 2001. Occurrence of mercury in some commercial fish species from the Magdalena and Meta Rivers in Colombia. Rev Aso Col Ictiol. 4: 15-9.

Cobaleda JA. 2005. Caracterización de la preparación y conocimiento de la publicación, acerca de los problemas de la salud humana con el uso y manejo de mercurio en la explotación de oro y el consumo de pescado proveniente de las fuentes hídricas, en el municipio de Caucasia, Antioquia. Protocolo del proyecto. Medellín: Universidad de Antioquia.

Gaudet C, Lingard S, Cureton P, Keenleyside K, Smith S, Raju G. 2005. Canadian Environmental Quality Guidelines for mercury. Water Air Soil Pollut. 80 (14): 1149-59. Disponible en: https://link.springer.com/ article/10.1007/BF01189777

Instituto de Investigaciones Ambientales del Pacifico (IIAP). 2014. Estudio de los efectos y perturbaciones fisico-química y biológica de fuentes hídricas presionadas por la actividad minera en el Distrito Minero del San Juan (Raspadura). Componente fauna íctica. Informe técnico.

Lancheros-Ascencio LJ. 2013. Contenido de mercurio en músculo de algunas especies ícticas de interés comercial presentes en ocho sitios de muestreo de la cuenca (baja, medio y alta) del río Magdalena. Bogotá: Planeación Ambiental y Administración de los Recursos y Universidad Militar Nueva Granada. 


\section{Bioetnia Volumen 14, 2017}

Lasso CA, Agudelo Córdoba E, Jiménez-Segura LF, Ramírez-Gil, H, Morales-Betancourt M,Ajiaco-Martínez $\mathrm{RE}$, et al. (eds.). 2011. I. Catálogo de los recursos pesqueros continentales de Colombia. Serie Editorial Recursos hidrobiológicos y pesqueros continentales de Colombia. Bogotá: Instituto de Investigación de Recursos Biológicos Alexander von Humboldt(IAvH); 715 pp.

Lebel J, Roulet M, Mergler D, Lucotte M, Larribe F. 1997. Fish diet and mercury expose in a riparian amazonian population. Water, Air Soil Pollution. 97 (1-2): 31-44. Disponible en: https://link.springer.com/ article/10.1007/BF02409642

Maldonado-Ocampo JA, Ortega-Lara A, Usma JS, Galvis G, Villa-Navarro FA, Vásquez L, et al. 2005. Peces de los Andes de Colombia. Guía de campo. Bogotá: Instituto de Investigación de Recursos Biológicos «Alexander von Humboldt»; 346 pp. Disponible en: http://awsassets.panda.org/downloads/peces de los andes de colombia.pdf

Maldonado-Ocampo JA, Usma JS, Villa-Navarro FA, Ortega-Lara A, Prada-Pedreros S, Jiménez LF, et al. 2012. Peces dulceacuícolas del Chocó Biogeográfico de Colombia. Bogotá: WWF Colombia, Instituto de Investigaciones de Recursos Biológicos "Alexander von Humboltd (IAvH)", Universidad del Tolima, Autoridad Nacional de Acuicultura y Pesca (AUNAP), Pontificia Universidad Javeriana; 400 pp. Disponible en: http://d2ouvy59p0dg6k.cloudfront.net/downloads/ peces_choco_baja.pdf

Mancera-Rodríguez NJ, Álvarez-León R. 2006. Estado del conocimiento de las concentraciones de mercurio y otros metales pesados en peces dulceacuícolas de Colombia. Acta Biol Colomb. 11 (1): 3-23. Disponible en: http:// www.scielo.org.co/pdf/abc/v11n1/v11n1a01.pdf

Marrugo J, Lans E, Benitez L. 2007. Hallazgo de mercurio en peces de la ciénaga de Ayapel, Córdoba, Colombia. Rev MVZ.12 (1): 878-86. Disponible en: http://www. scielo.org.co/pdf/mvz/v12n1/v12n1a03.pdf

Marrugo-Negrete J, Benítez N, Olivero-Verbel J. 2008. Distribution of mercury in several environmental com- partments in an aquatic ecosystem impacted by gold mining in northern Colombia. Arch Environ Contam Toxicol. 55: 305-16.

Ministerio de Salud y Protección Social de Colombia. 2012. Resolución $\mathrm{N}^{\circ} 000122$.

Olivero J, Johnson B. 2002. Contaminación con mercurio y salud pública en la costa atlántica colombiana. Biomédica. 22 (S1): 52-53.

Olivero-Verbel J, Caballero-Gallardo K, Turizo-Tapia A. 2015. Mercury in the gold mining district of San Martin de Loba, South of Bolivar(Colombia). Environ Sci Pollut Res. 22: 5895-907.

Plan de Desarrollo Municipio de Timbiquí 2012-2015. “Todos unidos por el Timbiquí que queremos". Timbiquí: PDMT.

Ramos CX, Estévez SL, Giraldo E. 2000. Nivel de contaminación por metilmercurio en la región de Mojana Bogotá: Departamento de Ingeniería Civil y Ambiental, Centro de Investigaciones en Ingeniería Ambiental (CIIA), Universidad de los Andes.

República de Colombia. 1984. Decreto 1594 de 1984. Usos del agua y residuos líquidos. Diario Oficial 36700 de julio 26 de 1984; 120 pp

Román-Valencia C. 1988. Clave taxonómica para la determinación de los peces nativos del departamento del Quindío subsistema alto río Cauca. Actual Biol. 17 (64): 107-13. Disponible en: https://www.researchgate.net/ publication/292132663 Clave taxonomica para la determinacion de peces nativos del departamento del_Quindio_subsistema_alto_rio_Cauca_Colombia

Román-Valencia C. 1990. Lista y distribución de peces en la cuenca media del río Atrato, Chocó, Colombia. Caldasia. 16(77): 201-7. Disponible en: https://revistas. unal.edu.co/index.php/cal/article/view/35528

Rivas-Lara T. 1993. La ictiofauna de la cuenca alta del río Atrato y su estado taxonómico. Tesis. Bogotá: Universidad Nacional.

WHO. 2000. Air quality guidelines for Europe. $2^{\text {nd }}$ ed. Copenhagen: World Health Organization, Regional Office for Europe. 288 pp. Disponible en: http:// www.euro.who.int/ data/assets/pdf file/0005/74732/ E71922.pdf 